\title{
A NOTE ON WEAK DIFFERENTIABILITY OF PETTIS INTEGRALS
}

\author{
M. EVANS MUNROE
}

Pettis ${ }^{1}$ raised the question whether or not separability of the range space implies almost everywhere weak differentiability of Pettis integrals. Phillips ${ }^{2}$ has given an example which answers this question in the negative. His construction is based on a sequence of orthogonal vectors in Hilbert space. We present here a different example of the same type of function. Our basic construction is that of a function defined to the space $C$. Using that function as a basis, we are able to give a specific construction of such a function defined to each member of a large class of Banach spaces.

1. Metric density properties of a non-dense perfect set. Let $B C[0,1]$ be a non-dense perfect set of measure one-half, and let $\bar{B}$ be its complement. $\bar{B}$ may be constructed by taking the sum of a set of open intervals classified as follows:

1 interval of length $1 / 4$,

2 intervals each of length $1 / 16$,

4 intervals each of length $1 / 64$,

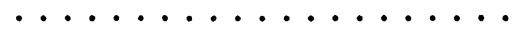

$2^{n-1}$ intervals each of length $1 / 2^{2 n}$,

We shall refer to the intervals of length $1 / 2^{2 n}$ as intervals of $\bar{B}$ of order $n$. We shall assume that each interval of $\bar{B}$ of order $n$ is the center portion of the space either between two intervals of $\bar{B}$ of lower order or between one such interval of $\bar{B}$ and an end point of the unit interval. These spaces we shall refer to as gaps of order $n$, and we shall denote such a gap by the symbol $G_{n}$. If $\bar{B}$ is constructed as noted above, then for each $n$, any two sets each of the form $G_{n} \cdot \bar{B}$ are congruent; hence we shall use $G_{n}$ to denote a gap of order $n$, and we shall not find it necessary to specify which one.

The following three lemmas are now obvious.

1.1. Lemma. $\left|\bar{B} \cdot G_{n}\right|=1 / 2^{2 n-1}$.

1.2. Lemma. $\left|G_{n}\right|=1 / 2^{n}+1 / 2^{2 n-1}$.

Presented to the Society, September 17, 1945; received by the editors July 18 , 1945, and, in revised form, August 28, 1945.

'See [3, p. 303]. Numbers in brackets refer to the references cited at the end of the paper.

2 See $[4$, p. 144]. 
1.3. Definition. If $I$ is any subinterval of $[0,1]$, we define

$$
\rho(I)=|\bar{B} \cdot I| /|I| \text {. }
$$

1.4. LemMa. $\rho\left(G_{n}\right)=1 /\left(1+2^{n-1}\right)$.

The following important lemma demonstrates a lower bound on the density function $\rho$. This lower bound may tend to zero as $|I|$ tends to zero, but it is independent of the location of $I$.

\subsection{LEMMA. If}

then

$$
|I| \geqq\left|G_{n}\right|=1 / 2^{n}+1 / 2^{2 n-1},
$$

$$
\rho(I)>1 /\left(2+2^{n-1}\right) \text {. }
$$

First suppose $|I|=\left|G_{n}\right|$. For all such $I$ the $G_{n}$ have minimum values for $\rho$; for if $I=G_{n}$ and then is moved a little to the right or left, some points of $B$ are excluded and only points of $\bar{B}$ are includedthus obviously increasing $\rho(I)$-until the interval of $\bar{B}$ is covered. However, this interval of $\bar{B}$ is of order at most $n-1$ and hence has measure at least $1 / 2^{2 n-2}$; thus so long as $I$ contains this interval of $\bar{B}$,

$$
\rho(I)>\frac{1 / 2^{2 n-2}}{1 / 2^{n}+1 / 2^{2 n-1}}=\frac{2}{1+2^{n-1}}=2 \rho\left(G_{n}\right) .
$$

If $I$ is moved on beyond this interval of $\bar{B}$ the above argument applies again by considering the movement in the reverse direction. Comparison with Lemma 1.4 now shows that Lemma 1.5 is established in case $|I|=\left|G_{n}\right|$.

Consider now the effect of increasing $|I|$. If $|I|=\left|G_{n}\right|$ and $I$ contains no interval of $\bar{B}$ of order less than or equal to $n-1$, then one end point of $I$ (let us assume it is the right-hand one) lies in the closure of such an interval of $\bar{B}$. Thus any small extension of $I$ to the right will (until the interval of $\bar{B}$ is covered) add to $I$ only points of $\bar{B}$, thus obviously increasing $\rho(I)$. If $I=G_{n}$ at the start, a small extension to the left will have the same effect. Otherwise an extension to the left may be regarded as a translation to the left and a subsequent extension to the right, and these cases have already been discussed. In case $I$ contains an interval of $\bar{B}$ of order not greater than $n-1$, we have $|\bar{B} \cdot I|>1 / 2^{2 n-2}$; thus if $|I| \leqq\left|G_{n-1}\right|=1 / 2^{n-1}+1 / 2^{2 n-8}$,

$$
\rho(I)>\frac{1 / 2^{2 n-2}}{1 / 2^{n-1}+1 / 2^{2 n-3}}=\frac{1}{2+2^{n-1}} .
$$

If $|I|>\left|G_{n-1}\right|$, the above argument may be repeated with $n-1$ sub- 
stituted for $n$, thereby obtaining an even larger lower bound for $\rho(I)$. This proves Lemma 1.5 for all cases.

1.6. Lemma. For all $I \subset[0,1]$,

$$
\rho(I)>|\bar{B} \cdot I|^{1 / 2} / 2^{5 / 2} \text {. }
$$

We shall prove Lemma 1.6 by showing that, for each $n$, the required inequality holds for

$$
1 / 2^{2 n+1}<|\bar{B} \cdot I| \leqq 1 / 2^{2 n-1} .
$$

This will cover all possibilities. For $|\bar{B} \cdot I|$ in this range, we consider first the case $|I|<\left|G_{n}\right|=1 / 2^{n}+1 / 2^{2 n-1}$. In this case

$$
\begin{aligned}
\frac{1}{\rho(I)} & =\frac{|I|}{|\bar{B} \cdot I|}<\frac{|I|}{1 / 2^{2 n+1}}<\frac{1 / 2^{n}+1 / 2^{2 n-1}}{1 / 2^{2 n+1}}=4+2^{n+1} \\
& =4+2^{3 / 2}\left[1 / 2^{2 n-1}\right]^{-1 / 2} \leqq 4+2^{3 / 2}|\bar{B} \cdot I|^{-1 / 2} .
\end{aligned}
$$

Considering now the case $|I| \geqq\left|G_{n}\right|$ (and assuming $|\bar{B} \cdot I|$ still in the same range) we have, using Lemma 1.5 and the above inequalities,

$$
1 / \rho(I)<2+2^{n-1}<4+2^{n+1} \leqq 4+2^{3 / 2}|\bar{B} \cdot I|^{-1 / 2} \text {. }
$$

Now for all $I C[0,1],|\bar{B} \cdot I| \leqq 1 / 2$; hence $|\bar{B} \cdot I|^{-1 / 2} \geqq 2^{1 / 2}$; hence $4 \leqq 2^{3 / 2}|\bar{B} \cdot I|^{-1 / 2}$. Combining this with the above results, we have

$$
1 / \rho(I)<4+2^{8 / 2}|\bar{B} \cdot I|^{-1 / 2} \leqq 2^{8 / 2}|\bar{B} \cdot I|^{-1 / 2},
$$

whence

$$
\rho(I)>|\bar{B} \cdot I|^{1 / 2} / 2^{5 / 2} \text {. }
$$

2. An approximately continuous function whose integral is nondifferentiable. For each $t \in B$ we define the function $f_{t}(x)$ for $x \in[0,1]$ as follows

$$
f_{t}(x)=\left\{\begin{array}{cc}
0 & \text { for } x \leqq t \text { or } x \in B, \\
|\bar{B} \cdot[t, x]|^{-8 / 4} & \text { for } x>t \text { and } x \in \bar{B} .
\end{array}\right.
$$

2.1. ThEOREM. For each $t \in B, f_{t}(x)$ is an integrable function of $x$, and for $x_{2} \geqq x_{1} \geqq t$,

$$
\int_{x_{1}}^{x_{2}} f_{t}(x) d x=4\left(\left|\bar{B} \cdot\left[t, x_{2}\right]\right|^{1 / 4}-\left|\bar{B} \cdot\left[t, x_{1}\right]\right|^{1 / 4}\right) .
$$

Let $z=|\bar{B} \cdot[t, x]|$. Since the intervals of $\bar{B}$ are dense in $[0,1]$, this defines $z$ as a strictly monotone function of $x$; hence $x$ is a singlevalued function of $z$, and we may write $f_{t}[x(z)]$. Now for $x>t$ and $x \in \bar{B}, d x=d z$; thus the function $z(x)$ is measure preserving over $\bar{B}$ 
- $[t, x]$ and hence maps $B \cdot[t, x]$ into a set of measure zero. Therefore, for almost all $z, f_{t}[x(z)]=z^{-3 / 4}$; and $d x=d z$ except where $f_{t}(x)=0$; so

$$
\int_{x_{1}}^{x_{2}} f_{l}(x) d x=\int_{z_{1}}^{z_{2}} z^{-3 / 4} d z=4\left(z_{2}^{1 / 4}-z_{1}^{1 / 4}\right) .
$$

2.2. Theorem. For $t \in B$, the function

$$
F_{t}(x)=\int_{t}^{x} f_{t}(u) d u
$$

is not differentiable with respect to $x$ at $x=t$.

Again letting $z=|\bar{B} \cdot[t, x]|$, and using Theorem 2.1 and Lemma 1.6, we have

Thus

$$
\begin{aligned}
\frac{F_{t}(x)}{x-t} & =\frac{4 z^{1 / 4}}{x-t}=4 z^{-3 / 4}\left(\frac{z}{x-t}\right)=4 z^{-3 / 4} \rho([t, x]) \\
& >4 z^{-3 / 4}\left(z^{1 / 2} / 2^{5 / 2}\right)=(4 z)^{-1 / 4} .
\end{aligned}
$$

$$
\limsup _{x \rightarrow t} \frac{F_{t}(x)}{x-t} \geqq \lim _{z \rightarrow 0}(4 z)^{-1 / 4}=\infty .
$$

In the next section we shall make further use of the functions $f_{t}(x)$ and their properties as shown in Theorems 2.1 and 2.2. We might note here, however, that $f_{t}(x)$ is approximately continuous at $t$ provided $\bar{B}$ has metric density zero at $t$. This is true for almost all $t$ in $B$; hence for such $t, f_{t}(x)$ furnishes a specific example of an approximately continuous function whose integral is not differentiable.

3. A Pettis integral in the space $C$ which is not almost everywhere weakly differentiable. We shall here define a function $\phi(x)$ from $[0,1]$ to the space $C$. Our notation will be as follows: For each $x \in[0,1]$, $\phi(x)$ stands for a continuous function on $[0,1]$; we denote this continuous function by $\phi_{x}(t)$. We shall define the functions $\phi_{x}(t)$ by defining a function $\phi(x, t)$ over the unit square and setting $\phi_{x}(t)=\phi(x, t)$. We first define $\phi(x, t)$ over a portion of the unit square as follows:

$$
\phi(x, t)=\left\{\begin{array}{cll}
0 & \text { for } & x \in B, \\
f_{t}(x) & \text { for } & t \in B .
\end{array}\right.
$$

Since $f_{t}(x)=0$ for $x \in B$, these statements are consistent.

3.1. Lemma. For a fixed $x, \phi(x, t)$ is continuous in $t$ over $B$.

This statement follows immediately from the fact that if one end 
point of $I$ is fixed, $|\bar{B} \cdot I|^{-3 / 4}$ is a continuous function of the other end point over any set such that $|I|$ is bounded away from zero. This latter restriction causes no difficulties here. If $x \in B, \phi(x, t)=0$; if $x \in \bar{B}$, dist $(x, B)>0$.

We now continue the definition of $\phi(x, t)$. For each $x \in \bar{B}$, let $\phi(x, t)$ be continued linearly over each interval of the set $t \in \bar{B}$. This completes the definition of $\phi(x, t)$ over the entire unit square, and it is clear that for each $x, \phi(x, t)$ is continuous in $t$ over $[0,1]$.

3.2. Theorem. $\phi(x)$ is integrable in the sense of Pettis. For each measurable set $E \subset[0,1]$, its integral over $E$ is the function

$$
\Phi_{E}(t)=\int_{E} \phi(u, t) d u .
$$

We show this by considering the functions $\phi^{(n)}(x)$ whose values are the continuous functions $\phi_{x}^{(n)}(t)=\phi^{(n)}(x, t)$ where

$$
\phi^{(n)}(x, t)=\left\{\begin{array}{cl}
\phi(x, t) & \text { for } \phi(x, t) \leqq n \\
n & \text { for } \phi(x, t)>n .
\end{array}\right.
$$

It is easily seen that for each $t \in[0,1]$, each $\phi^{(n)}(x, t)$ is bounded and continuous in $x$ over $\bar{B}$. Thus ${ }^{3}$ each $\phi^{(n)}(x)$ is weakly continuous over $\bar{B}$. Since $\phi^{(n)}(x)=\theta$ for $x \in B$, it is clear that each $\phi^{(n)}(x)$ is weakly measurable. Since $C$ is a separable space, it follows ${ }^{4}$ that each $\phi^{(n)}(x)$ is measurable. Now each $\phi^{(n)}(x)$ is bounded, hence Bochner integrable, hence Pettis integrable, therefore integrable with respect to each of the linear functionals $\gamma_{t}\left[\phi^{(n)}(x)\right]=\phi_{x}^{(n)}(t)$; thus

$$
\Phi^{(n)}(E)=\int_{E} \phi^{(n)}(u) d u
$$

is the continuous ${ }^{\mathbf{5}}$ function

$$
\Phi_{E}^{(n)}(t)=\int_{E} \phi^{(n)}(u, t) d u .
$$

Clearly for each $x$ and each $t$,

$$
\lim _{n \rightarrow \infty} \phi_{x}^{(n)}(t)=\phi_{x}(t)
$$

${ }^{3}$ See $[1$, p. 224, Theorem 8].

4 See [3, Theorem 1.1].

B An independent proof of continuity of $\Phi_{E}^{(n)}(t)$ is unnecessary. Since $\phi^{(n)}(x)$ is Pettis integrable, it is integrable to an element of $C_{\text {; }}$ and the set $\left\{\gamma_{t}\right\}$ of linear functionals defines this element uniquely. 
furthermore this approximation is monotone in $n$. Thus $\left\|\phi^{(n)}(x)-\phi(x)\right\|$ is bounded in $n$ for each $x$; hence $\phi^{\theta}(n)(x) \rightarrow \phi(x)$ weakly for each $x$. It now follows ${ }^{7}$ that $\phi(x)$ is Pettis integrable provided the sequence $\left\{\Phi^{(n)}(E)\right\}$ converges with respect to the norm in $C$; that is, provided $\left\{\Phi_{E}(n)(t)\right\}$ converges uniformly in $t$. We shall complete the proof of Theorem 3.2 by showing that for each measurable $E \subset[0,1]$

$$
\Phi_{E}(t)=\int_{E} \phi(u, t) d u
$$

exists for each $t$ and that this function is the uniform limit of the sequence $\left\{\Phi_{E^{(n)}}(t)\right\}$.

To show that $\Phi_{E}(t)$ exists is trivial. For $t \in B$, this follows from Theorem 2.1. For each $x, \phi(x, t)$ is extended linearly over each interval of $t \in \bar{B}$; hence for $t \in \bar{B}, \phi(x, t) \leqq \phi\left(x, t_{1}\right)+\phi\left(x, t_{2}\right)$ where $t_{1}$ and $t_{2}$ are each in $B$. This completes the proof of integrability.

Now with each $t \in[0,1]$ we associate two numbers $t_{1}$ and $t_{2}$ as follows: $t_{1}$ is the greatest number such that $t_{1} \in B$ and $t_{1} \leqq t ; t_{2}$ is the smallest number such that $t_{2} \in B$ and $t_{2} \geqq t$. Geometrically this means that if $t \in B, t_{1}=t=t_{2}$, while if $t \in \bar{B}, t_{1}$ and $t_{2}$ are the left and right points respectively of the interval of $\bar{B}$ in which $t$ is located.

Now for $t_{1} \leqq x<t_{2}, \phi(x, t) \leqq \phi\left(x, t_{1}\right)$ while for $x \geqq t_{2}, \phi(x, t) \leqq \phi\left(x, t_{2}\right)$. Thus for any given $t \in[0,1]$, it is possible to have $\phi(x, t)>n$ only for those values of $x$ for which either

$$
t_{1} \leqq x<t_{2} \text { and }\left|\bar{B} \cdot\left[t_{1}, x\right]\right|<n^{-4 / 8}
$$

or

$$
x \geqq t_{2} \text { and }\left|\bar{B} \cdot\left[t_{2}, x\right]\right|<n^{-4 / 3} .
$$

Outside these two intervals $\phi(x, t)-\phi^{(n)}(x, t)=0$; hence if we denote these intervals by $I_{1}$ and $I_{2}$, we have

$$
\begin{aligned}
\int_{E}\left[\phi(x, t)-\phi^{(n)}(x, t)\right] d x & \leqq \int_{I_{1}} \phi(x, t) d x+\int_{I_{2}} \phi(x, t) d x \\
& \leqq \int_{I_{1}} \phi\left(x, t_{1}\right) d x+\int_{I_{2}} \phi\left(x, t_{2}\right) d x \\
& <2 \int_{0}^{n-1 / 3} z^{-3 / d / d} d z=8 n^{-1 / 3} .
\end{aligned}
$$

- See $[1$, p. 224, Theorem 8].

${ }^{7}$ See $[3$, Theorem 4.1]. 
Thus, clearly, $\lim _{n \rightarrow \infty} \Phi_{E}^{(n)}(t)=\Phi_{E}(t)$ uniformly in $t$. This completes the proof of Theorem 3.2.

3.3. Theorem. If $x_{0} \in B, \Phi(x)=\int_{0}^{x} \phi(u) d u$ is not weakly differentiaable at $x_{0}$.

By Theorem 2.2, it fails to be differentiable at $x_{0}$ with respect to the linear functional $\gamma_{x_{0}}[\Phi(x)]=\Phi_{x}\left(x_{0}\right)$.

4. Extension to other spaces of continuous functions. The function $\phi(x)$ of $\S 3$ may be used as the basis for the construction of a large set of examples as follows:

4.1. THEOREM. If $\Omega$ is a compact metric space containing non-denumerably many points and if $C(\Omega)$ is the Banach space of all continuous functionals on $\Omega$, then there is a function $\psi(x)$ from the unit interval to $C(\Omega)$ such that $\psi(x)$ is Pettis integrable but $\Psi(E)=\int_{E} \psi(x) d x$ fails to be weakly differentiable on a set of positive measure.

Since $\Omega$ is non-denumerable, it contains a perfect set. This perfect set is a complete metric space which is dense in itself and hence contains a homeomorph $\Pi$ of the Cantor set $B .^{8}$

Let $B=h(\Pi)$ be the homeomorphic mapping of $\Pi$ into $B$. Then $h(\omega)$ is a continuous function defined over $\Pi$, assuming values between 0 and 1 , and assuming for some $\omega \in \Pi$ each value in the set $B$. Let $H(\omega)$ be a continuous extension ${ }^{9}$ of $h(\omega)$ over the whole of $\Omega$ with $0 \leqq H(\omega) \leqq 1$.

Now for each $t \in[0,1]$ we define

$$
K(t)=\varepsilon_{\omega}\{H(\omega)=t\} .
$$

It should be noted that although for some $t, K(t)$ may be vacuous, for each $t \in B, K(t)$ contains at least one point.

Referring back to the functions $\phi_{x}(t)$ of $\S 3$, we now define

$$
\psi_{x}(\omega)=\phi_{x}(t) \text { for } \omega \in K(t) .
$$

It follows from the continuity in $t$ of each function $\phi_{x}(t)$ and from the continuity of $H(\omega)$ that for each $x \in[0,1], \psi_{x}(\omega)$ is continuous over $\Omega$. For each $x \in[0,1]$ we now let $\psi(x)$ be the element $\psi_{x}(\omega)$ of $C(\Omega)$.

${ }^{8}$ See $[2$, p. 228]. The author is indebted to the referee for the suggestion that nondenumerability of $\Omega$ is sufficient to insure the existence of $I I$.

- See [2, p. 211]. In connection with our remark in the introduction that we have a specific construction applicable to the more general spaces, it should be noted that this extension theorem is not merely an existence proof. A definite formula for the extension is given. 
That $\psi(x)$ has the required properties may be seen as follows: To show integrability, we note that for each $\omega \in \Omega, \psi_{x}(\omega)$ is identical (as a function of $x$ ) with $\phi_{x}(t)$ for some $t \in[0,1]$. Then noting that Banach's criterion for weak convergence ${ }^{10}$ applies to the space $C(\Omega)$, we apply the proof of Theorem 3.2. To show non-differentiability, we note that for each $t \in B$ there is an $\omega \in \Omega$ such that $\psi_{x}(\omega)=\phi_{x}(t)$ for all $x \in[0,1]$. The proof of Theorem 3.3 then applies.

\section{REFERENCES}

1. S. Banach, Theorie des operations lineaires, Monografje Matematyczne, Warsaw, 1932.

2. C. Kuratowski, Topologie, I, Monografje Matematyczne, Warsaw, 1933.

3. B. J. Pettis, On integration in vector spaces, Trans. Amer. Math. Soc. vol. 44 (1938) pp. 277-304.

4. R. S. Phillips, Integration in a convex linear topological space, Trans. Amer. Math. Soc. vol. 47 (1940) pp. 114-145.

\section{U'NIVERSITY OF ILLINOIS}

${ }^{10}$ This is used in the proof of Theorem 3.2. See footnotes 3 and 6. 